\title{
Consumo, digestibilidade aparente e desempenho de vacas leiteiras alimentadas com concentrado processado de diferentes formas ${ }^{1}$
}

\section{Humberto Luiz Wernersbach Filho ${ }^{2}$, José Maurício de Souza Campos ${ }^{3}$, Anderson Jorge de Assis ${ }^{4}$, Sebastião de Campos Valadares Filho ${ }^{3}$, Augusto César de Queiroz ${ }^{3}$, Rilene Ferreira Diniz Valadares ${ }^{5}$, Rogério de Paula Lana ${ }^{3}$}

1 Parte da dissertação de mestrado do primeiro autor apresentada à Universidade Federal de Viçosa, UFV - MG

2 Mestre em Zootecnia.

${ }^{3}$ Departamento de Zootecnia - UFV.

${ }^{4}$ Formil Veterinária Ltda.

${ }^{5}$ Departamento de Veterinária - UFV.

RESUMO - O objetivo neste estudo foi avaliar o consumo, a digestibilidade aparente, a degradabilidade ruminal da MS e da $\mathrm{PB}$, a produção e a composição do leite de vacas leiteiras alimentadas com concentrado processado de diferentes formas Foram utilizadas 16 vacas da raça Holandesa, puras e mestiças, em dois níveis de produção de leite (30 e $20 \mathrm{~kg}$ de leite/dia), distribuídas em quatro quadrados latinos (dois para cada nível de produção). O experimento foi constituído por quatro períodos de 15 dias. As dietas experimentais, isoprotéicas, foram formuladas à base de silagem de milho e apresentavam as relações volumoso:concentrado 50:50 e 60:40 (na MS), respectivamente, para proporcionar produções de 30 e 20,0 kg de leite/dia A digestibilidade da MS não foi afetada pelo processamento, enquanto a da PB para vacas alimentadas com concentrado extrusado $(72,36 \%)$ foi menor que a do concentrado farelado, no nível de $50 \%$ de concentrado. A digestibilidade da fibra em detergente neutro nas vacas alimentadas com concentrado extrusado $(44,35 \%)$ foi menor no nível de $50 \%$ de concentrado A produção de leite foi maior nos animais que consumiram concentrado extrusado $(29,9 \mathrm{~kg} / \mathrm{dia})$ no nível de $50 \%$. Contudo, não houve diferença significativa no menor nível de concentrado na dieta. A composição do leite não diferiu entre os tratamentos em ambos os níveis de produção.

Palavras-chave: bovino, nutrição, ruminante

\section{Intake, apparent digestibility, and production of dairy cows fed concentrate processed in different forms}

\begin{abstract}
The objective of this trial was to study the effects of different forms of concentrate processing on intake, apparent total tract digestibility of nutrients, ruminal degradability of DM and CP, and milk yield and composition. Sixteen dairy cows, pure Holstein and crossbred, were blocked by production level (30.0 and $20.0 \mathrm{~kg} / \mathrm{day}$ ) and randomly assigned to two Latin squares with four periods of 15 days each. Diets were isonitrogenous and contained the following forage (corn silage):concentrate ratios: 50:50 and 60:40 (\% of DM) for cows yielding 30.0 and $20.0 \mathrm{~kg}$ of milk/day, respectively. Apparent total tract digestibility of DM was not affected by the different forms of concentrate processing while apparent CP digestibility for cows fed extruded concentrate (72.36\%) was lower than that for cows fed grounded concentrate at a forage:concentrate ratio of 50:50. Apparent total tract digestibility of neutral detergent fiber $(44,35 \%)$ was lower $(\mathrm{P}<0.05)$ for cows fed extruded concentrate at the lowest forage:concentrate. Milk yield was higher for cows fed the extruded diet $(29.9 \mathrm{~kg} / \mathrm{day})$, at the greatest concentrate level, but no significant differences were observed for cows at the lowest (60:40) concentrate level.
\end{abstract}

Key Words: bovine, nutrition, ruminant

\section{Introdução}

A procura por concentrados processados (peletizado, extrusado, floculado etc) para a alimentação do rebanho leiteiro com vistas à melhoria do desempenho produtivo dos sistemas de produção de leite tem crescido significativamente no Brasil. Entretanto, deve-se considerar que o processamento desses alimentos apresenta, no mercado brasileiro, custo maior que o de concentrados farelados.

O alimento concentrado é o componente de maior peso no custo final da alimentação do rebanho, correspondendo a $50 \%$ do custo total de produção. Por isso, a otimização de seu uso tem efeito direto na lucratividade do sistema de produção (Gomes, 2000). 
O processamento do concentrado consiste em uma alternativa para obtenção de maior produtividade com a mesma quantidade de alimento fornecida para o animal. No entanto, há dúvidas se o potencial produtivo de vacas em lactação afeta a utilização do alimento consumido.

Ressalta-se que o processamento de alimentos concentrados pode aumentar o custo da alimentação do rebanho, o que o tornaria inviável. Por isso, a avaliação de técnicas como o aumento da densidade energética e o processamento parcial de alguns constituintes desse alimento é importante pelo fato de que seu uso pode ter o mesmo efeito que o processamento total do alimento, reduzindo o custo da alimentação.

O consumo de MS é controlado por fatores fisiológicos, físicos e psicogênicos (Van Soest, 1994) e se correlaciona altamente com a produção de leite. Portanto, técnicas de processamento que possam inibir o efeito deletério de algum componente da ração são opções para a utilização de subprodutos agroindustriais, que, na maioria das vezes, podem ser encontrados por um preço muito menor que o do produto nobre.

A digestibilidade dos nutrientes está relacionada ao teor de energia e às características estruturais dos alimentos utilizados para ruminantes. Alguns fatores podem influenciar a digestibilidade in vivo, entre eles o nível de consumo, o efeito associativo entre os alimentos, o processamento do alimento e a espécie animal (Kitessa et al., 1999).

O desempenho animal depende dos nutrientes fornecidos na dieta e da atividade metabólica em suas estruturas químicas. A degradação da proteína do alimento no rúmen gera compostos nitrogenados para a síntese de proteína microbiana, que apresenta excelente perfil de aminoácidos, cujo conteúdo é capaz de suprir grande parte do requerimento de proteína do animal em produção (Valadares Filho, 1995).

Broderick et al. (1991) relataram que, sob elevados níveis de produção, ocorre aumento nas necessidades protéicas e, para atender a estas condições, é necessário maximizar a eficiência de síntese de proteína microbiana, de modo que parte da proteína dietética consumida não seja degradada no rúmen.

Quando o pagamento ao produtor é feito com base no teor de gordura e proteína do leite, observa-se grande influência dos alimentos processados na porcentagem e no perfil de gordura do leite. A suplementação com alimentos concentrados extrusados pode diminuir o teor de gordura do leite e alterar sua composição (Whitlock et al., 2002).

Este trabalho foi conduzido visando avaliar o efeito de diferentes tipos de processamento do alimento concentrado sobre o consumo, a digestibilidade aparente dos nutrientes, a produção e a composição do leite de vacas leiteiras.

\section{Material e Métodos}

O experimento foi conduzido na Unidade de Ensino Pesquisa e Extensão em Gado de Leite do Departamento de Zootecnia da Universidade Federal de Viçosa, durante o período de outubro a dezembro de 2001.

Foram utilizadas 16 vacas da raça Holandesa, puras e mestiças, com pesos médios de 552 e $547 \mathrm{~kg}$, respectivamente, distribuídas equitativamente em quatro quadrados latinos (dois para cada nível de produção de leite; 30 e $20 \mathrm{~kg}$ de leite/ dia), balanceados pela duração e ordem da lactação.

O experimento foi constituído por quatro períodos de 15 dias, sendo os oito primeiros para adaptação às dietas e os demais para coleta de dados.

As dietas experimentais, isoprotéicas, foram formuladas à base de silagem de milho e apresentavam as relações volumoso:concentrado 50:50 e 60:40 na MS, para proporcionar produções de 30 e $20 \mathrm{~kg}$ de leite/dia, respectivamente. Os tratamentos consistiram de quatro alimentos concentrados sob as formas farelada (CF), peletizada (CP), extrusada (CE) e com alto teor de energia (CAE), parcialmente processada, com apenas milho e soja extrusados em sua composição.

As dietas foram formuladas para atender às exigências nutricionais dos animais, segundo recomendações do NRC (1989).

Nas Tabelas 1 e 2 são apresentadas as proporções dos ingredientes utilizados na formulação dos concentrados e as composições bromatológicas médias das rações e da silagem de milho. As composições bromatológicas das dietas são descritas nas Tabelas 3 e 4 .

Os animais foram manejados em baias individuais, tipo "Tie Stall”, onde receberam alimentação ad libitum, duas vezes ao dia, às 8 e $17 \mathrm{~h}$. Diariamente, foram feitas pesagens das quantidades das dietas fornecidas e das sobras de cada tratamento, para determinação do consumo. Diariamente, efetuou-se o monitoramento do consumo, a fim de manter as sobras de alimento em torno de $10 \%$, com base na MS. No momento da alimentação, durante o período experimental, foram feitas amostragens das dietas e das sobras, que foram acondicionadas em sacos plásticos e congeladas para análises posteriores.

As vacas foram ordenhadas mecanicamente duas vezes ao dia e sua produção de leite foi registrada por meio de dispositivo eletrônico acoplado à ordenhadeira. Foi coletada amostra de leite (aproximadamente $300 \mathrm{~mL}$ ) no $12^{\circ}$ dia, nas ordenhas da manhã e da tarde, elaborando-se uma amostra composta para análise dos teores de proteína, gordura, extrato seco total e extrato seco desengordurado. 
Tabela 1- Composição percentual dos ingredientes utilizados nos concentrados farelado (CF), peletizado (CP), extrusado (CE) e com alto teor de energia (CAE) $(\%$ na MS)

Table 1 - Ingredient composition of ground (GC), pelleted (PC), extruded (EC), and high energy (HEC) concentrates, \%DM

\begin{tabular}{|c|c|c|c|c|}
\hline \multirow[b]{2}{*}{$\begin{array}{l}\text { Ingrediente } \\
\text { Ingredient }\end{array}$} & \multicolumn{4}{|c|}{$\begin{array}{l}\text { Concentrado } \\
\text { Concentrate }\end{array}$} \\
\hline & $\begin{array}{l}\mathrm{CF} \\
G C\end{array}$ & $\begin{array}{l}\mathrm{CP} \\
P C\end{array}$ & $\begin{array}{l}\mathrm{CE} \\
E C\end{array}$ & $\begin{array}{l}\mathrm{CAE} \\
H E C\end{array}$ \\
\hline Milho & 45 & 45 & 45 & 50 \\
\hline $\begin{array}{l}\text { Corn } \\
\text { Milho extrusado moído } \\
\text { Ground extruded corn }\end{array}$ & - & - & - & 10 \\
\hline $\begin{array}{l}\text { Farelo de trigo } \\
\text { Wheat meal }\end{array}$ & 7,3 & 7,3 & 7,3 & - \\
\hline $\begin{array}{l}\text { Soja integral extrusada } \\
\text { Extruded integral soybean }\end{array}$ & - & - & - & 10 \\
\hline $\begin{array}{l}\text { Farelo soja } \\
\text { Soybean meal }\end{array}$ & 31,9 & 31,9 & 31,9 & 24,45 \\
\hline $\begin{array}{l}\text { Farelo de arroz } \\
\text { Rice meal }\end{array}$ & 10,8 & 10,8 & 10,8 & - \\
\hline $\begin{array}{l}\text { Uréia } \\
\text { Urea }\end{array}$ & 1,2 & 1,2 & 1,2 & 1,0 \\
\hline $\begin{array}{l}\text { Fosfato bicálcico } \\
\text { Dicalcium phosphate }\end{array}$ & 0,38 & 0,38 & 0,38 & 1,63 \\
\hline $\begin{array}{l}\text { Calcário } \\
\text { Limestone }\end{array}$ & 2,0 & 2,0 & 2,0 & 0,63 \\
\hline $\begin{array}{l}\text { Sal comum } \\
\text { Salt }\end{array}$ & 0,6 & 0,6 & 0,6 & 0,7 \\
\hline $\begin{array}{l}\text { Flor de enxofre } \\
\text { Sulfur flower }\end{array}$ & 0,19 & 0,19 & 0,19 & - \\
\hline $\begin{array}{l}\text { Premix ruminante } \\
\text { Ruminant premix }\end{array}$ & 0,8 & 0,8 & 0,8 & 1,0 \\
\hline $\begin{array}{l}\text { Premix tamponante } \\
\text { Buffer }\end{array}$ & - & - & - & 0,6 \\
\hline
\end{tabular}

Tabela 2 - Teores médios de MS, MO, PB, EE, fibra em detergente neutro corrigida para cinzas e proteína (FDNcp), FDA e LIG, obtidos nos concentrados farelado (CF), peletizado (CP), extrusado (CE) e com alto teor de energia (CAE) e na silagem de milho

Table 2 - $\quad$ Average contents of DM, OM, CP, EE, NDF, ADF, and LIG of ground $(G C)$, pelleted $(P C)$, extruded (EC), and high energy (HEC) concentrates and corn silage

\begin{tabular}{|c|c|c|c|c|c|}
\hline \multirow[b]{2}{*}{$\begin{array}{l}\text { Componente } \\
\text { Component }\end{array}$} & \multicolumn{4}{|c|}{$\begin{array}{l}\text { Concentrado } \\
\text { Concentrate }\end{array}$} & \multirow[b]{2}{*}{$\begin{array}{c}\text { Silagem de milho } \\
\text { Corn silage }\end{array}$} \\
\hline & $\begin{array}{l}\mathrm{CF} \\
G C\end{array}$ & $\begin{array}{l}\mathrm{CP} \\
\mathrm{PC}\end{array}$ & $\begin{array}{l}\mathrm{CE} \\
E C\end{array}$ & $\begin{array}{l}\mathrm{CAE} \\
H E C\end{array}$ & \\
\hline $\begin{array}{l}\text { MS (\%) } \\
D M\end{array}$ & 89,01 & 88,96 & 89,94 & 89,88 & 32,23 \\
\hline $\begin{array}{l}\mathrm{MO}(\%) \\
O M\end{array}$ & 87,89 & 87,50 & 88,73 & 88,80 & 31,39 \\
\hline $\begin{array}{l}\mathrm{PB}^{1} \\
C P\end{array}$ & 26,68 & 25,03 & 25,30 & 25,53 & 8,07 \\
\hline $\begin{array}{l}\mathrm{EE}^{1} \\
\mathrm{FDNcp}^{1}\end{array}$ & $\begin{array}{c}4,44 \\
18,77\end{array}$ & $\begin{array}{c}4,20 \\
24,73\end{array}$ & $\begin{array}{c}4,40 \\
19,29\end{array}$ & $\begin{array}{c}4,58 \\
15,95\end{array}$ & $\begin{array}{c}3,68 \\
48,26\end{array}$ \\
\hline $\begin{array}{l}N D F \\
\mathrm{FDA}^{1} \\
A D F\end{array}$ & 8,64 & 9,88 & 9,05 & 7,40 & 30,18 \\
\hline $\mathrm{LIG}^{1}$ & 1,68 & 1,83 & 1,90 & 1,22 & 4,02 \\
\hline
\end{tabular}

$1 \%$ na matéria seca (\% dry matter).
Tabela 3 - Teores médios de MS, MO, PB, EE, CHO FDN, CNF FDA, LIG e NDT em vacas alimentadas com dietas contendo $50 \%$ de concentrado farelado (CF), peletizado (CP), extrusado (CE) ou com alto teor de energia, parcialmente processado (CAE)

Table 3 - $\quad$ Average contents of DM, OM, CP, EE, CHO, NDF, NFC, ADF and Lig, TDN for cows fed diets with $50 \%$ of ground (GC), pelleted (PC), extruded (EC), and high energy (HEC) concentrates and corn silage

\begin{tabular}{|c|c|c|c|c|}
\hline \multirow[t]{2}{*}{$\begin{array}{l}\text { Componente } \\
\text { Item }\end{array}$} & \multicolumn{4}{|c|}{$\begin{array}{c}50 \% \text { de concentrado } \\
50 \% \text { of concentrate }\end{array}$} \\
\hline & $\begin{array}{l}\mathrm{CF} \\
G C\end{array}$ & $\begin{array}{l}\mathrm{CP} \\
P C\end{array}$ & $\begin{array}{l}\mathrm{CE} \\
E C\end{array}$ & $\begin{array}{l}\mathrm{CAE} \\
\mathrm{HEC}\end{array}$ \\
\hline $\begin{array}{l}\text { MS }(\%) \\
D M\end{array}$ & 60,62 & 60,60 & 61,09 & 61,06 \\
\hline $\begin{array}{l}\mathrm{MO}^{1} \\
O M\end{array}$ & 98,07 & 97,88 & 98,03 & 98,10 \\
\hline $\begin{array}{l}\mathrm{PB}^{1} \\
C P\end{array}$ & 17,38 & 16,55 & 16,69 & 16,80 \\
\hline $\mathrm{EE}^{1}$ & 4,06 & 3,94 & 4,04 & 4,13 \\
\hline $\mathrm{CHO}^{1}$ & $\begin{array}{l}76,64 \\
33,52\end{array}$ & $\begin{array}{l}77,39 \\
36,50\end{array}$ & $\begin{array}{l}78,38 \\
33,78\end{array}$ & $\begin{array}{l}77,17 \\
32,11\end{array}$ \\
\hline NDF & & & & \\
\hline $\begin{array}{l}\mathrm{CNF}^{1} \\
N F C\end{array}$ & 43,12 & 40,90 & 44,61 & 45,07 \\
\hline $\begin{array}{l}\mathrm{FDA}^{1} \\
A D F\end{array}$ & 19,41 & 20,03 & 19,62 & 18,79 \\
\hline $\begin{array}{l}\mathrm{LIG}^{1} \\
\mathrm{NDT}^{1}\end{array}$ & $\begin{array}{c}2,85 \\
55,35\end{array}$ & $\begin{array}{c}2,93 \\
55,97\end{array}$ & $\begin{array}{c}2,96 \\
58,41\end{array}$ & $\begin{array}{c}2,62 \\
65,45\end{array}$ \\
\hline$T D N$ & & & & \\
\hline
\end{tabular}

As análises de MS, MM, compostos nitrogenados (N), EE, FDN, FDA e LIG foram realizadas segundo metodologias descritas por Silva \& Queiroz (2002).

Foram coletadas amostras de fezes do $8^{\circ}$ ao $14^{\circ}$ dia do período experimental, uma vez por dia, com início (tempo 0 ) às $8 \mathrm{~h}$ do 8 o dia e, nos dias subseqüentes, em intervalos de $2,4,6,8,10$ e 12 horas em relação ao primeiro dia de coleta. Em seguida, estimou-se a excreção fecal por meio da relação consumo de fibra em detergente ácido indigestível (FDAi) dividido por sua concentração fecal. A digestibilidade dos nutrientes foi calculada de acordo com Lippke et al. (1986), utilizando-se a FDAi. Aproximadamente $250 \mathrm{mg}$ das amostras de sobras de alimentos, sobras e fezes foram incubados no interior do rúmen por 144 horas e, posteriormente, fervidos em detergente ácido, considerando-se o resíduo o teor de FDAi.

As degradabilidades ruminais da proteína bruta (PB) foram estimadas pela técnica do saco de náilon (Ørskov \& McDonald, 1979). Foram pesados $5 \mathrm{~g}$ de cada alimento concentrado e colocados em sacos de náilon com dimensão de 7 x $14 \mathrm{~cm}$ e poros de, aproximadamente, $50 \mathrm{~mm}$. Procurou-se manter a relação de $20 \mathrm{mg}$ de $\mathrm{MS} / \mathrm{cm}^{2}$ de área de superfície dos sacos, conforme recomendações de Kirkpatrick \& Kennelly (1987). A incubação dos sacos de náilon no rúmen foi realizada em duas vacas lactantes mantidas sob mesmo 
Tabela 4 - Teores médios de MS, MO, PB, EE, CHO, FDN, CNF, FDA, LIG e NDT em vacas alimentadas com dietas contendo $40 \%$ de concentrado farelado (CF), peletizado (CP), extrusado (CE) ou com alto teor de energia, parcialmente processado (CAE)

Table 4 - Average contents of DM, OM, CP, EE, CHO, NDF, NFC, ADF, LIG, and TDN for cows fed diets with $40 \%$ of ground (GC), pelleted ( $P C$ ), extruded (EC), and high energy (HEC) concentrates

\begin{tabular}{|c|c|c|c|c|}
\hline \multirow[t]{2}{*}{$\begin{array}{l}\text { Componente } \\
\text { Item }\end{array}$} & \multicolumn{4}{|c|}{$\begin{array}{c}40 \% \text { de concentrado } \\
40 \% \text { of concentrate }\end{array}$} \\
\hline & $\begin{array}{l}\mathrm{CF} \\
G C\end{array}$ & $\begin{array}{l}\mathrm{CP} \\
P C\end{array}$ & $\begin{array}{l}C E \\
E C\end{array}$ & $\begin{array}{l}\text { CAE } \\
H E C\end{array}$ \\
\hline $\operatorname{MS}(\%)(D M)$ & 54,94 & 54,92 & 55,31 & 55,29 \\
\hline $\mathrm{MO}^{1}(O M)$ & 97,94 & 97,78 & 97,90 & 97,96 \\
\hline $\mathrm{PB}^{1}(C P)$ & 15,51 & 14,85 & 14,96 & 15,05 \\
\hline $\mathrm{EE}^{1}$ & 3,98 & 3,89 & 3,97 & 4,04 \\
\hline $\mathrm{CHO}^{1}$ & 78,44 & 79,04 & 79,83 & 78,87 \\
\hline $\mathrm{FDN}^{1}(N D F)$ & 36,46 & 38,85 & 36,67 & 35,34 \\
\hline $\mathrm{CNF}^{1}(N F C)$ & 41,97 & 40,19 & 43,16 & 43,53 \\
\hline $\mathrm{FDA}^{1}(A D F)$ & 21,56 & 22,06 & 21,73 & 21,07 \\
\hline $\mathrm{LIG}^{1}$ & 3,08 & 3,14 & 3,17 & 2,90 \\
\hline $\mathrm{NDT}^{1}(T D N)$ & 53,20 & 60,05 & 56,86 & 59,04 \\
\hline
\end{tabular}

$1 \%$ na matéria seca (in dry matter basis).

programa alimentar (silagem de milho e concentrado comercial na relação 55:45), conforme os intervalos recomendados pelo NRC (2001): 0, 2, 4, 8, 16, 24 e 48 horas. Os parâmetros de cinética de degradação da PB foram estimados utilizando-se o modelo descrito por Ørskov \& McDonald (1979), segundo a equação: $\mathrm{Dg}=\mathrm{a}+\mathrm{b}\left(1-\mathrm{e}^{\mathrm{ct}}\right)$, em que a representa uma estimativa da fração instantaneamente solúvel; b, a fração insolúvel, mas potencialmente fermentável; c, a taxa de degradação; e t, o tempo. A degradabilidade efetiva foi calculada pela equação: $\mathrm{DE}=\mathrm{a}+(\mathrm{b} \times \mathrm{kd}) /(\mathrm{kd}+\mathrm{kp})$, em que kd é a taxa de degradação no rúmen e kp, a taxa de passagem no rúmen, calculada pela equação: $\mathrm{Kp}=2,904+$ $1,375 \mathrm{X}_{1}-0,020 \mathrm{X}_{2}$, em que $\mathrm{X}_{1}$ é o consumo de MS (\%PV)e $\mathrm{X}_{2}$, a porcentagem de concentrado proposta pelo NRC (2001).

Os carboidratos totais (CHO) foram calculados conforme proposto por Sniffen et al. (1992):

$$
\mathrm{CHO}=100-(\% \mathrm{~PB}+\% \mathrm{EE}+\% \mathrm{CINZAS})
$$

e os carboidratos não-fibrosos (CNF), pela seguinte equação:

$$
\mathrm{CNF}=\mathrm{CHO}-\mathrm{FDN}
$$

Os nutrientes digestíveis totais (NDT) foram estimados segundo o NRC (2001):

$$
\mathrm{NDT}(\%)=\mathrm{PBD}+\mathrm{FDND}+\mathrm{CNFD}+2,25 * \mathrm{EED}
$$

em que $\mathrm{PBD}=$ proteína bruta digestível; $\mathrm{FDND}$ = fibra em detergente neutro digestível; CNFD = carboidratos nãofibrosos digestíveis; e EED = extrato etéreo digestível.

$\mathrm{O}$ teor de nitrogênio total do leite, analisado pelo método micro Kjeldahl, foi multiplicado pelo fator 6,38, para determinação do teor de PB. A determinação dos extratos seco total e desengordurado do leite foi feita pelo método de Behmer (1984) e a de gordura, segundo Pregnolatto \& Pregnolatto (1985).

A produção de leite corrigida para 3,5\% de gordura (PLCG) foi calculada utilizando-se a fórmula de Sklan et al. (1992):

$$
\begin{gathered}
P L C G=(0,432+0,1625 \times \% \text { gordura do leite }) \times \text { produção } \\
\text { de leite em kg/dia. }
\end{gathered}
$$

Os dados obtidos foram submetidos à análise de variância, comparando-se as médias pelo teste de Newman Keuls, a 5\% de significância, utilizando-se o programa SAEG, versão 7.1. (UFV, 1997).

\section{Resultados e Discussão}

Os consumos médios diários de MS, MO, $\mathrm{PB}, \mathrm{EE}, \mathrm{CHO}$, FDN, CNF e NDT por vacas alimentadas com 50 e $40 \%$ de concentrado na MS, respectivamente, são apresentados nas Tabelas 5 e 6 .

$\mathrm{O}$ consumo de MS não foi afetado $(\mathrm{P}>0,05)$ pela forma de processamento do concentrado, dentro de cada nível de produção, em relação a rações fareladas.

$\mathrm{O}$ fornecimento do concentrado com alto teor de energia e parcialmente processado promoveu menor $(\mathrm{P}<0,05)$ consumo de MS. O fator que condicionou esse menor consumo de MS - e conseqüentemente de PB, EE e ND - não foi detectado neste experimento. Resultado semelhante foi encontrado por Shabi et al. (1999), que observaram menor consumo de MS por vacas leiteiras alimentadas com milho extrusado em relação ao concentrado farelado, indicando que análises mais profundas devem ser feitas em dietas com maior densidade energética contendo com alimentos parcialmente processados.

Os coeficientes de digestibilidade aparente de MS, MO, $\mathrm{PB}, \mathrm{EE}, \mathrm{CHO}, \mathrm{FDN}$ e CNF observados nas vacas alimentadas com 50 e $40 \%$ de concentrado na dieta (com base na MS), respectivamente, são apresentados nas Tabelas 7 e 8.

As técnicas de processamento total do concentrado não afetaram $(\mathrm{P}>0,05)$ os coeficientes de digestibilidade da MS do concentrado farelado utilizado na dieta com $50 \%$ de concentrado. Entre as dietas com 50\% de concentrado, maior coeficiente de digestibilidade da MS $(\mathrm{P}<0,05)$ foi obtido apenas naquela com concentrado de alto teor de energia parcialmente processado, provavelmente em razão do menor consumo de MS, que promove maior coeficiente de digestibilidade, em razão do maior tempo de retenção da digesta.

No nível de inclusão de $50 \%$ de concentrado nas dietas, o coeficiente de digestibilidade da FDN foi menor $(\mathrm{P}<0,05)$ para o concentrado extrusado. Os coeficientes de 
Tabela 5 - Consumos médios diários de MS, MO, PB, EE, CHO, FDN, CNF e NDT em vacas alimentadas com dietas contendo $50 \%$ de concentrado farelado (CF), peletizado (CP), extrusado (CE) ou com alto teor de energia, parcialmente processado (CAE)

Table 5 - $\quad$ Average daily intakes of DM, OM, CP, EE, CHO, NDF, NSC, and TDN for cows fed diets with $50 \%$ of ground (GC), pelleted $(P C)$, extruded (EC), and high energy (HEC) concentrates

\begin{tabular}{|c|c|c|c|c|c|}
\hline \multirow[t]{3}{*}{$\begin{array}{l}\text { Item } \\
\text { Item }\end{array}$} & \multicolumn{4}{|c|}{$\begin{array}{c}\text { Forma de processamento do concentrado } \\
\text { Concentrate processing }\end{array}$} & \multirow[t]{3}{*}{$\mathrm{CV} \%$} \\
\hline & $\mathrm{CF}$ & $\mathrm{CP}$ & $\mathrm{CE}$ & $\mathrm{CAE}$ & \\
\hline & $G C$ & $P C$ & $E C$ & HEC & \\
\hline \multicolumn{6}{|c|}{$\begin{array}{l}\text { Consumo }(\mathrm{kg} / \mathrm{dia}) \\
\text { Intake }(\mathrm{kg} / \text { day })\end{array}$} \\
\hline $\operatorname{MS}(D M)$ & $20,07 \mathrm{a}$ & $19,90 \mathrm{a}$ & $20,15 \mathrm{a}$ & $16,50 \mathrm{~b}$ & 10,33 \\
\hline $\mathrm{MO}(O M)$ & $16,77 \mathrm{a}$ & $16,93 a$ & $17,07 \mathrm{a}$ & $13,75 b$ & 10,15 \\
\hline $\mathrm{PB}(C P)$ & $3,38 \mathrm{a}$ & $3,14 \mathrm{a}$ & $3,37 \mathrm{a}$ & $2,61 b$ & 10,03 \\
\hline $\mathrm{EE}$ & $0,77 \mathrm{a}$ & $0,74 \mathrm{a}$ & $0,54 b$ & $0,59 \mathrm{~b}$ & 12,50 \\
\hline $\mathrm{CHO}$ & $14,57 \mathrm{a}$ & $15,01 \mathrm{a}$ & $15,64 \mathrm{a}$ & $12,05 b$ & 11,40 \\
\hline $\mathrm{FDN}(N D F)$ & $6,25 \mathrm{a}$ & $6,75 \mathrm{a}$ & $6,42 \mathrm{a}$ & $4,95 b$ & 11,34 \\
\hline $\mathrm{CNF}(N F C)$ & $5,43 \mathrm{a}$ & $4,66 \mathrm{a}$ & $6,16 \mathrm{a}$ & $4,89 \mathrm{a}$ & 22,59 \\
\hline NDT $(T D N)$ & $11,11 \mathrm{a}$ & $11,14 \mathrm{a}$ & $11,77 \mathrm{a}$ & $10,80 \mathrm{a}$ & 16,24 \\
\hline \multicolumn{6}{|c|}{$\begin{array}{c}\text { Consumo }(\% \mathrm{PV}) \\
\text { Intake }(\% \quad B W)\end{array}$} \\
\hline $\operatorname{MS}(D M)$ & $3,59 \mathrm{a}$ & $3,54 \mathrm{a}$ & $3,59 \mathrm{a}$ & $3,02 b$ & 12,27 \\
\hline FDN $(N D F)$ & $1,12 b$ & $1,20 \mathrm{~b}$ & $1,14 b$ & $0,90 \mathrm{a}$ & 9,18 \\
\hline
\end{tabular}

Médias seguidas de letras iguais na mesma linha não diferem $(P>0,05)$ pelo teste de Newman Keuls.

Means followed by the same letter in a row do not differ $(P<0.05)$ by Newman Keuls test. $\mathrm{CV}=$ Coeficiente de variação (Coefficient of variation).

Tabela 6 - Consumos médios diários de MS, MO, PB, EE, CHO, FDN, CNF e NDT obtidos em vacas alimentadas com dietas contendo $40 \%$ de concentrado farelado (CF), peletizado (CP), extrusado (CE) ou com alto teor de energia, parcialmente processado (CAE)

Table 6 - Average daily intakes of DM, OM, CP, EE, CHO, NDF, NSC and total digestible nutrients (TDN) obtained for cows fed diets with $40 \%$ of ground $(G C)$, pelleted (PC), extruded (EC), and high energy (HEC) concentrates

\begin{tabular}{|c|c|c|c|c|c|}
\hline \multirow[t]{3}{*}{$\begin{array}{l}\text { Item } \\
\text { Item }\end{array}$} & \multicolumn{4}{|c|}{$\begin{array}{l}\text { Forma de processamento do concentrado } \\
\text { Concentrate processing }\end{array}$} & \multirow[t]{3}{*}{ CV \% } \\
\hline & $\mathrm{CF}$ & $\mathrm{CP}$ & $\mathrm{CE}$ & CAE & \\
\hline & $G C$ & $P C$ & $E C$ & HEC & \\
\hline & \multicolumn{5}{|c|}{$\begin{array}{l}\text { Consumo }(\mathrm{kg} / \mathrm{dia}) \\
\text { Intake }(\mathrm{kg} / \text { dav })\end{array}$} \\
\hline $\operatorname{MS}(D M)$ & $18,40 \mathrm{a}$ & $18,20 \mathrm{a}$ & $17,78 \mathrm{a}$ & $16,70 \mathrm{a}$ & 8,08 \\
\hline $\mathrm{MO}(O M)$ & $14,94 \mathrm{a}$ & $15,32 \mathrm{a}$ & $15,05 \mathrm{a}$ & $13,70 \mathrm{a}$ & 8,50 \\
\hline $\mathrm{PB}(C P)$ & $2,76 \mathrm{a}$ & $2,63 \mathrm{a}$ & $2,68 \mathrm{a}$ & $2,41 b$ & 7,67 \\
\hline $\mathrm{EE}$ & $0,70 \mathrm{a}$ & $0,68 \mathrm{a}$ & $0,51 \mathrm{c}$ & $0,63 b$ & 8,30 \\
\hline $\mathrm{CHO}$ & $14,14 \mathrm{a}$ & $14,31 \mathrm{a}$ & $14,24 \mathrm{a}$ & $12,67 \mathrm{a}$ & 9,09 \\
\hline FDN (NDF) & $6,00 \mathrm{a}$ & $6,52 \mathrm{a}$ & $5,86 \mathrm{a}$ & $5,22 b$ & 8,90 \\
\hline $\mathrm{CNF}(N F C)$ & $4,91 \mathrm{a}$ & $5,13 \mathrm{a}$ & $5,35 \mathrm{a}$ & $4,98 \mathrm{a}$ & 15,56 \\
\hline \multirow[t]{2}{*}{ NDT (TDN) } & $9,79 \mathrm{a}$ & $10,93 \mathrm{a}$ & $10,11 \mathrm{a}$ & $9,86 \mathrm{a}$ & 11,58 \\
\hline & \multicolumn{5}{|c|}{$\begin{array}{c}\text { Consumo }(\% \mathrm{PV}) \\
\text { Intake }(\% B W)\end{array}$} \\
\hline $\mathrm{MS}(D M)$ & $3,37 \mathrm{a}$ & $3,29 \mathrm{a}$ & $3,27 \mathrm{a}$ & $3,09 \mathrm{a}$ & 7,20 \\
\hline FDN $(N D F)$ & $1,09 \mathrm{~b}$ & $1,18 \mathrm{~b}$ & $1,07 \mathrm{~b}$ & $0,97 \mathrm{a}$ & 7,40 \\
\hline
\end{tabular}

Médias seguidas de letras iguais na mesma linha não diferem $(P>0,05)$ pelo teste de Newman Keuls.

Means followed by the same letter in a row do not differ $(P<0.05)$ by Newman Keuls test. $\mathrm{CV}=$ Coeficiente de variação (Coefficient of variation).
Tabela 7 - Digestibilidades aparentes médias de MS, MO, PB, EE, $\mathrm{CHO}, \mathrm{FDN}$ e CNF obtidas em vacas alimentadas com dietas contendo $50 \%$ de concentrado farelado (CF), peletizado $(\mathrm{CP})$, extrusado (CE) ou com alto teor de energia, parcialmente processado (CAE)

Table 7 - Average apparent digestibilities of DM OM, CP, EE, CHO, NDF and NSC, for cows fed diet with $50 \%$ of ground (GC), pelleted $(P C)$, extruded $(E C)$, and high energy (HEC) concentrates

\begin{tabular}{|c|c|c|c|c|c|}
\hline \multirow[t]{2}{*}{$\begin{array}{l}\text { Item } \\
\text { Item }\end{array}$} & \multicolumn{4}{|c|}{$\begin{array}{c}\text { Forma de processamento do concentrado } \\
\text { Concentrate processing }\end{array}$} & \multirow[t]{2}{*}{$\mathrm{CV} \%$} \\
\hline & $\begin{array}{l}\text { CF } \\
G C\end{array}$ & $\begin{array}{l}\mathrm{CP} \\
\mathrm{PC}\end{array}$ & $\begin{array}{l}C E \\
E C\end{array}$ & $\begin{array}{c}\text { CAE } \\
\text { HEC }\end{array}$ & \\
\hline $\operatorname{MS}(D M)$ & $72,31 \mathrm{a}$ & $70,31 \mathrm{a}$ & $67,57 \mathrm{a}$ & $76,32 b$ & 5,22 \\
\hline $\mathrm{MO}(O M)$ & $74,39 \mathrm{ab}$ & $72,58 b$ & $69,94 \mathrm{~b}$ & $77,59 a$ & 4,89 \\
\hline $\mathrm{PB}(C P)$ & $78,16 \mathrm{a}$ & $76,43 \mathrm{ab}$ & $72,36 b$ & $78,04 \mathrm{a}$ & 5,32 \\
\hline $\mathrm{EE}$ & $84,31 \mathrm{a}$ & $86,80 \mathrm{a}$ & $84,35 \mathrm{a}$ & $84,96 a$ & 4,46 \\
\hline $\mathrm{CHO}(\mathrm{CHO})$ & $72,29 b$ & $71,12 b$ & $69,53 b$ & $76,61 \mathrm{a}$ & 4,91 \\
\hline $\mathrm{FDN}(N D F)$ & $52,36 a$ & $50,75 \mathrm{a}$ & $44,35 b$ & $57,84 a$ & 10,56 \\
\hline $\mathrm{CNF}(\mathrm{NSC})$ & $78,72 \mathrm{a}$ & $86,23 \mathrm{a}$ & $81,02 \mathrm{a}$ & $84,16 \mathrm{a}$ & 13,09 \\
\hline
\end{tabular}

Médias seguidas de letras iguais na mesma linha não diferem $(P>0,05)$ pelo teste de Newman Keuls.

Means followed by the same letter in a row do not differ $(P<0.05)$ by Newman Keuls test. $\mathrm{CV}=$ Coeficiente de variação (Coefficient of variation).

Tabela 8 - Digestibilidades aparentes médias da MS, MO, PB, EE, $\mathrm{CHO}, \mathrm{FDN}$ e CNF obtidas em vacas alimentadas com dietas contendo $40 \%$ de concentrado farelado (CF), peletizado $(\mathrm{CP})$, extrusado (CE) ou com alto teor de energia, parcialmente processado (CAE)

Table 8 - Average apparent digestibilities of DM OM, CP, EE, CHO, NDF, and NSC for cows fed diet with $40 \%$ of ground (GC), pelleted $(P C)$, extruded (EC), and high energy (HEC) concentrates

\begin{tabular}{|c|c|c|c|c|c|}
\hline \multirow[t]{3}{*}{ Item } & \multicolumn{4}{|c|}{$\begin{array}{c}\text { Forma de processamento do concentrado } \\
\text { Concentrate processing }\end{array}$} & \multirow[t]{3}{*}{$\mathrm{CV} \%$} \\
\hline & $\mathrm{CF}$ & $\mathrm{CP}$ & $\mathrm{CE}$ & CAE & \\
\hline & GC & $P C$ & $E C$ & HEC & \\
\hline $\operatorname{MS}(D M)$ & $67,99 a$ & $71,01 \mathrm{a}$ & $69,92 \mathrm{a}$ & $73,16 a$ & 4,93 \\
\hline $\mathrm{MO}(O M)$ & $69,29 a$ & $72,85 \mathrm{a}$ & $71,80 \mathrm{a}$ & $74,56 \mathrm{a}$ & 5,03 \\
\hline $\mathrm{PB}(C P)$ & $70,76 \mathrm{a}$ & $75,51 \mathrm{a}$ & $71,47 \mathrm{a}$ & $75,39 a$ & 5,76 \\
\hline $\mathrm{EE}$ & $80,30 \mathrm{a}$ & $86,91 \mathrm{a}$ & $85,12 \mathrm{a}$ & $83,83 \mathrm{a}$ & 5,31 \\
\hline $\mathrm{CHO}$ & $70,03 \mathrm{a}$ & $72,50 \mathrm{a}$ & $72,62 \mathrm{a}$ & $74,24 \mathrm{a}$ & 4,58 \\
\hline FDN $(N D F)$ & $50,92 \mathrm{a}$ & $55,40 \mathrm{a}$ & $50,76 \mathrm{a}$ & $56,46 \mathrm{a}$ & 10,07 \\
\hline $\mathrm{CNF}(\mathrm{NSC})$ & $68,07 \mathrm{a}$ & $76,66 a$ & $79,25 \mathrm{a}$ & $79,00 \mathrm{a}$ & 13,09 \\
\hline
\end{tabular}

Médias seguidas de letras iguais na mesma linha não diferem $(P>0,05)$ pelo teste de Newman Keuls.

Means followed by the same letter in a row do not differ $(P<0.05)$ by Newman Keuls test. $\mathrm{CV}=$ Coeficiente de variação (Coefficient of variation).

digestibilidade da PB para os concentrados farelado e com alto teor de energia, parcialmente processado, foram maiores $(\mathrm{P}<0,05)$ que os do concentrado extrusado.

Não houve diferença $(\mathrm{P}>0,05)$ significativa nos coeficientes de digestibilidade entre as dietas com menor nível de concentrado, possivelmente em decorrência da forma de processamento do concentrado, que não influenciou os consumos de MS, MO, CHO, CNF e NDT, e da pequena diferença observada para os teores de PB, EE e FDN, que não foram suficientes para alterar a digestibilidade dessas frações. 
Os coeficientes de cinética de degradação ruminal da MS e PB encontram-se na Tabela 9.

A taxa de degradação ruminal da MS foi numericamente maior para a RE, indicando que, nesse tratamento, os nutrientes poderiam estar mais disponíveis aos microrganismos ruminais. O mesmo comportamento pode ser observado para a taxa de degradação da PB, uma vez que os concentrados peletizado e extrusado apresentaram taxas de degradação da proteína numericamente maiores que as dos outros tratamentos. Com isso, observa-se que a degradabilidade efetiva da proteína (DEPB) para os concentrados peletizado e extrusado foi numericamente maior em detrimento à dos concentrados farelado e com alto teor de energia parcialmente processado.

Considerando-se os conceitos do NRC (2001), a taxa de passagem foi maior para o nível de $50 \%$ de concentrado $(0,0681$ e 0,0657 , para 50 e $40 \%$ de concentrado na dieta, respectivamente). Portanto, a solubilidade dos nutrientes pode estar altamente relacionada ao suprimento da demanda nutricional. Desta forma, quando se utilizam alimentos capazes de fornecer quantidade superior de carboidratos e proteínas, em mesma faixa de consumo e taxa de passagem, a demanda microbiana por nutrientes pode ser atendida, podendo ocorrer maior produção de proteína microbiana, que é finalmente aproveitada pelo animal.

Tabela 9 - Parâmetros da cinética de degradação ruminal e degradabilidade efetiva (DE) da MS e PB em vacas alimentadas com dietas contendo $50 \%$ de concentrado farelado (CF), peletizado (CP), extrusado (CE) ou com alto teor de energia, parcialmente processado (CAE)

Table 9 - Kinetic parameters of ruminal and effective degradability (ED) of DM and CP degradation for cows fed diet with $50 \%$ of ground $(G C)$, pelleted ( $P C)$, extruded (EC), and high energy (HEC) concentrates

\begin{tabular}{|c|c|c|c|c|c|}
\hline \multirow{3}{*}{$\begin{array}{l}\text { Tratamento } \\
\text { Treatment }\end{array}$} & \multirow{2}{*}{\multicolumn{3}{|c|}{$\begin{array}{l}\text { Fração } \\
\text { Fraction }\end{array}$}} & \multirow{3}{*}{$\begin{array}{l}\mathrm{DE}(\%) \\
E D(\%)\end{array}$} & \multirow[t]{3}{*}{$\mathrm{R}^{2 \%}$} \\
\hline & & & & & \\
\hline & a $(\%)$ & $\mathrm{b}(\%)$ & $\mathrm{Kd} / \mathrm{h}$ & & \\
\hline & \multicolumn{5}{|c|}{$\begin{array}{l}\text { Degradação da matéria seca } \\
\text { Dry matter degradation }\end{array}$} \\
\hline $\mathrm{CF}(G C)$ & 18,80 & 64,52 & 0,063 & 50,46 & 97,90 \\
\hline $\mathrm{CP}(P C)$ & 28,86 & 56,48 & 0,070 & 58,30 & 94,60 \\
\hline $\mathrm{CE}(E C)$ & 10,02 & 67,20 & 0,060 & 70,74 & 94,20 \\
\hline \multirow[t]{2}{*}{ CAE (HEC) } & 23,90 & 64,55 & 0,064 & 56,81 & 93,60 \\
\hline & \multicolumn{5}{|c|}{$\begin{array}{l}\text { Degradação da proteína bruta } \\
\text { Crude protein degradation }\end{array}$} \\
\hline $\mathrm{CF}(G C)$ & 33,87 & 52,85 & 0,056 & 58,25 & 97,30 \\
\hline $\mathrm{CP}(P C)$ & 37,00 & 46,24 & 0,151 & 69,43 & 90,50 \\
\hline $\mathrm{CE}(E C)$ & 23,74 & 54,69 & 0,664 & 73,62 & 94,30 \\
\hline CAE (HEC) & 29,94 & 63,59 & 0,043 & 56,10 & 90,00 \\
\hline
\end{tabular}

a - Fração prontamente solúvel (High soluble fraction).

b - Fração insolúvel parcialmente degradável (Partially degradable insoluble fraction ).

kd - Taxa de degradação da fração b (Degradation rate of fraction $b$ ).
Consta na Tabela 10 uma comparação entre as exigências nutricionais (NRC,1989) e os consumos dos alimentos, apresentados na Tabela 5. Considerou-se, para efeito dos cálculos, uma vaca lactante com peso corporal de $550 \mathrm{~kg}$ produzindo $30 \mathrm{~kg} / \mathrm{dia}$ de leite. Em todos os tratamentos, houve déficit de NDT para os animais, apesar de a variação no peso corporal ter sido $-0,06 ; 0,34$ e 0,47 ( $\mathrm{kg} / \mathrm{dia}$ ), para os tratamentos $\mathrm{CF}, \mathrm{CP}$ e $\mathrm{CE}$, respectivamente.

A produção e composição do leite para vacas alimentadas com 50 e $40 \%$ de concentrado, com base na MS, são apresentadas nas Tabelas 11 e 12 , respectivamente.

A dieta contendo concentrado extrusado no nível de $50 \%$ na MS promoveu maior $(\mathrm{P}<0,05)$ produção de leite, com aumento de $9,96 \%$ em relação àquela contendo concentrado farelado (Tabela 11), podendo compensar o custo do processamento do concentrado, tornando-se uma opção para alimentação de vacas leiteiras com alto potencial produtivo. Uma das principais causas para esse aumento na produção de leite pode ter sido a taxa de degradação da MS e, principalmente, de proteína bruta encontrada nesse tratamento, indicando que o processamento total do concentrado por extrusão aumentou a eficiência alimentar, uma vez que os consumos dos nutrientes (PB, EE e NDT) nos tratamentos com os concentrados peletizado e extrusado não diferiram estatisticamente daquele registrado para o concentrado farelado.

A menor produção da dieta contendo concentrado parcialmente processado com alto teor de energia para o nível de $50 \%$ de concentrado na MS pode ser explicada pelo menor consumo dos nutrientes (PB, EE NDT), principalmente o de PB (Tabela 5). Em decorrência do menor consumo de MS e da maior digestibilidade dos nutrientes, o consumo de NDT

Tabela 10 - Exigências (E) e consumos observados (C) de PB e NDT em vacas com $550 \mathrm{~kg}$ de PV produzindo $30 \mathrm{~kg}$ de leite/dia com $3,5 \%$ de gordura alimentadas com dietas contendo $50 \%$ de concentrado farelado (CF), peletizado $(\mathrm{CP})$, extrusado $(\mathrm{CE})$ ou com alto teor de energia, parcialmente processado (CAE)

Table 10 - Requeriments (R) and observed intake (I) of CP and TDN for cows with $550 \mathrm{~kg}$ of BW yielding $30 \mathrm{~kg}$ of milk/day with $3.5 \%$ fat anf fed diet with $50 \%$ of ground $(G C)$, pelleted $(P C)$, extruded (EC), and high energy (HEC) concentrates

\begin{tabular}{|c|c|c|c|c|}
\hline \multirow[t]{2}{*}{ Item } & \multicolumn{2}{|c|}{$\begin{array}{l}\mathrm{PB}^{1} \\
C P^{l} \\
\end{array}$} & \multicolumn{2}{|c|}{$\begin{array}{c}\mathrm{NDT}^{1} \\
T D N^{l}\end{array}$} \\
\hline & $\mathrm{E}(R)^{2}$ & $\mathrm{C}(I)$ & $\mathrm{E}(R)^{2}$ & $\mathrm{C}(I)$ \\
\hline $\mathrm{CF}(G C)$ & 3,27 & 3,38 & 14,54 & 11,11 \\
\hline $\mathrm{CP}(P C)$ & 3,27 & 3,14 & 14,54 & 11,14 \\
\hline $\mathrm{CE}(E C)$ & 3,27 & 3,37 & 14,54 & 11,77 \\
\hline CAE (HEC) & 3,27 & 2,61 & 14,54 & 10,80 \\
\hline
\end{tabular}

${ }^{1} \mathrm{~kg} / \mathrm{dia}(\mathrm{kg} / \mathrm{day})$.

2 NRC (1989). 
Tabela 11 - Composição média e produções médias diárias de leite sem $(P L)$ e com correção para $3,5 \%$ de gordura (PLCG), obtidas em vacas alimentadas com dietas contendo $50 \%$ de concentrado farelado (CF), peletizado (CP), extrusado (CE) ou com alto teor de energia, parcialmente processado (CAE)

Table 11 - Milk yield (MY), 3.5\% fat corrected milk (FCM) and milk composition for cows fed diet with $50 \%$ of ground (GC), pelleted $(P C)$, extruded $(E C)$, and high energy (HEC) concentrates

\begin{tabular}{|c|c|c|c|c|c|}
\hline \multirow[t]{2}{*}{ Forn } & \multicolumn{4}{|c|}{$\begin{array}{l}\text { ma de processamento do concentrado } \\
\text { Concentrate processing }\end{array}$} & \multirow[t]{2}{*}{$\mathrm{CV} \%$} \\
\hline & $\begin{array}{l}\text { CF } \\
G C\end{array}$ & $\begin{array}{l}\mathrm{CP} \\
\mathrm{PC}\end{array}$ & $\begin{array}{l}\mathrm{CE} \\
\mathrm{EC}\end{array}$ & $\begin{array}{l}\text { CAE } \\
\text { HEC }\end{array}$ & \\
\hline $\begin{array}{l}\mathrm{PL}(\mathrm{kg} / \mathrm{vaca} / \mathrm{dia}) \\
M Y(\mathrm{~kg} / \text { day })\end{array}$ & $27,21 b$ & $27,64 b$ & $29,92 \mathrm{a}$ & $24,99 \mathrm{c}$ & 7,47 \\
\hline $\begin{array}{l}\text { PLCG (kg/vaca/dia) } \\
F C M(\mathrm{~kg} / \text { day })\end{array}$ & $25,93 \mathrm{a}$ & $25,54 \mathrm{a}$ & $26,83 \mathrm{a}$ & $23,89 a$ & 11,70 \\
\hline $\begin{array}{l}\text { Gordura }(\%) \\
\text { Fat }(\%)\end{array}$ & $3,20 \mathrm{a}$ & $3,04 \mathrm{a}$ & $2,82 \mathrm{a}$ & $3,19 \mathrm{a}$ & 22,63 \\
\hline $\begin{array}{l}\text { Proteína (\%) } \\
\text { Protein (\%) }\end{array}$ & $2,67 \mathrm{a}$ & $2,68 \mathrm{a}$ & $2,72 \mathrm{a}$ & $2,52 \mathrm{a}$ & 6,99 \\
\hline $\begin{array}{l}\text { Extrato seco } \\
\text { total }(\%) \\
\text { Total solids (\%) }\end{array}$ & $11,56 \mathrm{a}$ & $11,52 \mathrm{a}$ & $11,27 \mathrm{a}$ & $11,50 \mathrm{a}$ & 6,31 \\
\hline $\begin{array}{l}\text { Extrato seco } \\
\text { desengordurado (\%) } \\
\text { Solids non fat } \% \text { ) }\end{array}$ & $8,36 \mathrm{a}$ & $8,48 \mathrm{a}$ & $8,44 \mathrm{a}$ & $8,31 \mathrm{a}$ & 2,37 \\
\hline
\end{tabular}

Médias seguidas de letras iguais na mesma linha não diferem $(P>0,05)$ pelo teste Newman Keuls.

Means followed by the same letter in a row do not differ $(P<0.05)$ by Newman Keuls test. $\mathrm{CV}=$ Coeficiente de variação (coefficient of variation).

Tabela 12 - Composição média e produções médias diárias do leite sem $(P L)$ e com correção para $3,5 \%$ de gordura (PLCG), obtidas em vacas alimentadas com dietas contendo $40 \%$ de concentrado farelado (CF), peletizado (CP), extrusado (CE) ou com alto teor de energia, parcialmente processado (CAE)

Table 12 - Milk yield (MY), 3.5\% fat corrected milk (FCM) and milk composition for cows fed diet with $40 \%$ of ground (GC), pelleted $(P C)$, extruded $(E C)$, and high energy (HEC) concentrates

\begin{tabular}{|c|c|c|c|c|c|}
\hline \multirow[t]{2}{*}{ Forn } & \multicolumn{4}{|c|}{$\begin{array}{l}\text { rma de processamento do concentrado } \\
\text { Form of processing of concentrate }\end{array}$} & \multirow[t]{2}{*}{$\mathrm{CV} \%$} \\
\hline & $\begin{array}{l}\text { CF } \\
\text { GC }\end{array}$ & $\begin{array}{l}\mathrm{CP} \\
P C\end{array}$ & $\begin{array}{l}C E \\
E C\end{array}$ & $\begin{array}{l}\text { CAE } \\
\text { HEC }\end{array}$ & \\
\hline $\begin{array}{l}\mathrm{PL}(\mathrm{kg} / \mathrm{vaca} / \mathrm{dia}) \\
M Y(\mathrm{~kg} / \text { day })\end{array}$ & $18,43 \mathrm{a}$ & $18,06 \mathrm{a}$ & $18,75 \mathrm{a}$ & $17,49 a$ & 6,69 \\
\hline $\begin{array}{l}\text { PLCG }(\mathrm{kg} / \mathrm{vaca} / \text { dia }) \\
F C M(\mathrm{~kg} / \text { day })\end{array}$ & $18,89 \mathrm{a}$ & $20,77 \mathrm{a}$ & $19,17 \mathrm{a}$ & $18,69 \mathrm{a}$ & 10,54 \\
\hline $\begin{array}{l}\text { Gordura }(\%) \\
\text { Fat }(\%)\end{array}$ & $3,73 a$ & $4,48 \mathrm{a}$ & $3,75 \mathrm{a}$ & $3,96 a$ & 16,74 \\
\hline $\begin{array}{l}\text { Proteína }(\%) \\
\text { Protein }(\%)\end{array}$ & $3,03 \mathrm{a}$ & $3,05 \mathrm{a}$ & $3,68 \mathrm{a}$ & $3,06 \mathrm{a}$ & 22,26 \\
\hline $\begin{array}{l}\text { Extrato seco } \\
\text { total (\%) } \\
\text { Total solids (\%) }\end{array}$ & $12,80 \mathrm{a}$ & $13,37 \mathrm{a}$ & $12,84 \mathrm{a}$ & $12,93 \mathrm{a}$ & 4,90 \\
\hline $\begin{array}{l}\text { Extrato seco } \\
\text { desengordurado (\%) }\end{array}$ & $9,07 \mathrm{a}$ & $8,89 a$ & $9,09 \mathrm{a}$ & $8,97 \mathrm{a}$ & 2,71 \\
\hline
\end{tabular}

Médias seguidas de letras iguais na mesma linha não diferem $(P>0,05)$ pelo teste Newman Keuls.

Means followed by the same letter in a row do not differ $(P<0.05)$ by Newman Keuls test $\mathrm{CV}=$ Coeficiente de variação (coefficient of variation). não foi afetado, indicando que, em dietas energeticamente densas, pode ser necessário o aumento da concentração protéica do concentrado para que a quantidade de $\mathrm{PB}$ consumida seja compensada pelo menor consumo de MS, proporcionando melhor resposta no desempenho animal.

Não houve diferença estatística na produção de leite com o nível de $40 \%$ de concentrado na MS, provalmente porque os tratamentos não afetaram o consumo de nutrientes, mantendo o aporte para a síntese do leite (Tabela 12). Os animais do tratamento com $40 \%$ de concentrado produziram, em média, $18,18 \mathrm{~kg} / \mathrm{vaca} /$ dia e, como a média nacional de produção de leite/vaca/dia é muito menor, o processamento do concentrado parece não ser viável para rebanhos de médio ou baixo potencial produtivo.

Não foram observados efeitos do processamento sobre a composição do leite, independentemente do nível de produção.

\section{Conclusões}

Os processamentos total (peletização e extrusão) e parcial (soja integral extrusada) do concentrado não interferem na produção de leite de vacas com médio potencial de produção (18,0 kg/dia), o que torna sua utilização desvantajosa na alimentação desses animais. Entretanto, o processamento total do concentrado por extrusão promoveu aumento na produção de leite em vacas com alto potencial de produção $(30,0 \mathrm{~kg} / \mathrm{dia})$, indicando que seu uso é uma decisão de ordem econômica.

\section{Agradecimento}

À Universidade Federal de Viçosa, ao Departamento de Zootecnia da UFV e à Empresa Agroceres Nutrição Animal, pelo apoio.

\section{Literatura Citada}

BEHMER, M.L.A. Tecnologia do leite: produção, industrialização e análise. 13.ed. São Paulo: Nobel, 1984. p.100-108.

BRODERICK G.A.; WALLACE, R.J.; ØRSKOV, E.R. Control of rate and extend of protein degradation. In: TSUDA, T.; SASAKI, Y.; KAWASHIMA, R. (Eds.) Physiological aspects of digestion and metabolism in ruminants. New York: Academic Press, 1991. p.542-592.

GOMES, T.G. Economia da produção de leiteira. Belo Horizonte: Itambé, 2000. 132p.

GUILLAUME, B.; OTTERBY, D.E.; STERN, M.D. et al. Raw or extruded soybeans and rumen-protected methionine and lysine in alfafa-based diets for dairy cows. Journal of Dairy Science, v.74, p.1912, 1991.

KITESSA, S.; FLINN, P.C.; IRISH, G.G. Comparison of methods used to predict the in vivo digestibility of feeds in ruminants. Australian Journal Agriculture Research, v. 50, p.825-841, 1999. 
KIRKPATRICK, B.K.; KENNELY, J.J. In situ degradability of protein and dry matter from single protein sources and from a total diet. Journal of Animal Science, v.65, p.567-576, 1987.

LIPPKE, H.; ELLIS, W.C.; JACOBS, B.F. Recovery of indigestible fiber from feces of sheep and cattle on forage diets. Journal of Dairy Science, v.69, n.2, p.403-412, 1986.

NATIONAL RESEARCH COUNCIL - NRC. Nutrient requirements of dairy cattle. 7.ed. Washington, D.C.: National Academy Press, 2001. 381p.

NATIONAL RESEARCH COUNCIL - NRC. Nutrient requirements of dairy cattle. 6.ed. Washington, D.C.: National Academy Press, 1989. $158 \mathrm{p}$.

ØRSKOV, E.R.; McDONALD, I. The estimation of protein degradability in the rumen from incubation measurements weighed according to rate of passage. Journal of Agriculture Science, v.92, p.499-503, 1979.

PREGNOLATTO, W.; PREGNOLATO, N.P. Normas analíticas do Instituto Adolfo Lutz: métodos químicos e físicos para análise de alimentos. 3.ed. São Paulo, 1985. v.1, p.533.

SHABI, Z.; BRUCKENTAL, I.; ZAMWELL, S. et al. Effects of extrusion of grain and feeding frequency on rumen fermentation, nutrient digestibility, and milk yield and composition in dairy cows. Journal of Dairy Science, v.82, p.1252-1260, 1999 .

SILVA, D.J.; QUEIROZ, A.C. Análise de alimentos (Métodos químicos e biológicos). Viçosa, MG: Universidade Federald e Viçosa, 2002. 235p.

SKLAN, D.; ASHKENAZ, R.; BRAUN, A. et. al. Fatty acids, calciun soaps of fatty acids and cottonseeds fed to high yielding cows. Journal of Dairy Science, v.75, n.9, p.2463-2472, 1992.
SNIFFEN, C.J.; O'CONNOR, J.D.; Van SOEST, P.J. et al. A net carbohydrate and protein system for evaluating cattle diets. II. Carbohydrate and protein availability. Journal of Animal Science, v.70, n.11, p.3562-3577, 1992.

UNIVERSIDADE FEDERAL DE VIÇOSA - UFV. Sistema de Análises Estatísticas e Genéticas - SAEG. Versão 7.1. Viçosa, MG, 1997a. 150p. (Manual do usuário)

VALADARES FILHO, S.C. Eficiência da síntese de proteína microbiana, degradação ruminal e digestibilidade intestinal da proteína bruta, em bovinos. In: SIMPÓSIO INTERNACIONAL SOBRE EXIGÊNCIAS NUTRICIONAIS DE RUMINANTES. 1995, Viçosa, MG. Anais... Viçosa, MG: Universidade Federal de Viçosa, 1995. p.355-388.

Van SOEST, P.J. Nutricional ecology of the ruminant. 2.ed. Ithaca: Cornell, 1994. 476p.

WHITLOCK, L.A.; SCHINGOETHE D.J.; HIPPEN, A.R. et al. Fish oil and extruded soybeans fed in combination increase conjugated linoleic acids in milk of dairy cows more than when fed separately. Journal of Dairy Science, v. 85 , p.234-243, 2002 .

Recebido: $12 / 08 / 04$ Aprovado:08/08/05 